Supporting Information for

\title{
Dual-Atomic Cu Sites for Electrocatalytic CO Reduction to $\mathrm{C}_{2+}$ Products
}

Si Li, Anxiang Guan, Chao Yang, Chen Peng, Ximeng Lv, Yali Ji, Yueli Quan, Qihao Wang, Lijuan

Zhang, * Gengfeng Zheng*

Laboratory of Advanced Materials, Department of Chemistry and Shanghai Key Laboratory of

Molecular Catalysis and Innovative Materials, Shanghai 200438, China.

*Corresponding authors: Lijuan Zhang, Gengfeng Zheng

Email address: zhanglijuan@fudan.edu.cn (L.Z.),gfzheng@fudan.edu.cn(G.Z.) 


\section{Methods}

Chemicals. $\mathrm{Cu}(\mathrm{II})$ acetate monohydrate $\left(\mathrm{Cu}\left(\mathrm{CH}_{3} \mathrm{COO}\right)_{2} \cdot \mathrm{H}_{2} \mathrm{O}\right.$, Sigma), Nickel(II) acetate tetrahydrate $\left(\mathrm{Ni}\left(\mathrm{CH}_{3} \mathrm{COO}\right)_{2} \cdot 4 \mathrm{H}_{2} \mathrm{O}\right.$, Sigma), L-glutamic acid $\left(\mathrm{HOOCCH}\left(\mathrm{NH}_{2}\right)\left(\mathrm{CH}_{2}\right)_{2} \mathrm{COOH}\right.$, Aldrich), trimesic acid $\left(\mathrm{C}_{6} \mathrm{H}_{3}(\mathrm{COOH})_{3}\right.$, Aldrich), ethanol $\left(\mathrm{C}_{2} \mathrm{H}_{5} \mathrm{OH}\right.$, Aldrich), hydroxylamine hydrochloride $\left(\left(\mathrm{NH}_{3} \mathrm{OH}\right) \mathrm{Cl}\right.$, Sigma), glucose $\left(\mathrm{C}_{6} \mathrm{H}_{6} \mathrm{O}_{6}\right.$, Aldrich), nickel(II) chloride hexahydrate $\left(\mathrm{NiCl}_{2} \cdot 6 \mathrm{H}_{2} \mathrm{O}\right.$, Sigma), dicyandiamide $\left(\left(\mathrm{H}_{2} \mathrm{~N}\right)_{2} \mathrm{CNCN}\right.$, Aldrich $)$.

Synthesis of $\mathrm{Cu}$ MOF, $\mathrm{Cu}_{1.4} \mathrm{Ni}$ MOF and CuNi MOF. In a typical synthesis, $2.092 \mathrm{~g}$ of copper(II) acetate monohydrate and $0.771 \mathrm{~g}$ of L-glutamic acid were dispersed in $500 \mathrm{~mL}$ of deionized (DI) water and dissolved by sonication to obtain solution A. Then, $1.160 \mathrm{~g}$ of trimesic acid was dissolved in a solution consisting of $50 \mathrm{~mL}$ of ethanol and $450 \mathrm{~mL}$ of DI water to form solution B. Next, the solution A and solution B were mixed and kept stirring for two hours. The mixture was centrifugalized and then washed with DI water for several times. After being dried in vacuum oven overnight, $\mathrm{Cu}(\mathrm{BTC})\left(\mathrm{H}_{2} \mathrm{O}\right)_{3}$ (i.e., the $\mathrm{Cu} \mathrm{MOF}$ precursor) was obtained. The $\mathrm{Cu}_{1.4} \mathrm{Ni}$ MOF and CuNi MOF were also prepared under the similar conditions, except for the addition of $0.523 \mathrm{~g}(2.1 \mathrm{mmol})$ or $1.306 \mathrm{~g}(5.25 \mathrm{mmol})$ of nickel(II) acetate tetrahydrate, respectively, to keep the total molar numbers of $\mathrm{Cu}$ and $\mathrm{Ni}$ as $10.5 \mathrm{mmol}$.

Synthesis of dual $\mathbf{C u}, \mathrm{Cu}_{1.4} \mathbf{N i}$, and $\mathrm{CuNi}$ SACs. In brief, $0.1 \mathrm{~g}$ of precursors $(\mathrm{Cu} \mathrm{MOF}$, $\mathrm{Cu}_{1.4} \mathrm{Ni} \mathrm{MOF}$, or $\mathrm{CuNi} \mathrm{MOF}$ ) and $1 \mathrm{~g}$ of dicyandiamide were mixed and milled thoroughly. The sample was transferred into a quartz tube and annealed at $800^{\circ} \mathrm{C}$, and kept for 150 min with a heating rate of $3{ }^{\circ} \mathrm{C} \cdot \mathrm{min}^{-1}$ under Ar atmosphere. The product obtained after annealing was denoted as $\mathrm{Cu} \mathrm{N} / \mathrm{C}$. Ultimately, the $\mathrm{Cu}$ N/C was leached with oxygen-saturated 5\% HClovernight to obtain 
the final sample as the dual $\mathrm{Cu}$ SAC. The $\mathrm{Cu}_{1.4} \mathrm{Ni}$ and $\mathrm{CuNi}$ SACs were also synthesized under the similar conditions.

Synthesis of Ni SAC. In brief, $5 \mathrm{mg}$ of nickel(II) chloride hexahydrate puratrem, $690 \mathrm{mg}$ of hydroxylamine hydrochloride, and $144 \mathrm{mg}$ of glucose were dissolved in $40 \mathrm{~mL}$ of DI water and 40 $\mathrm{mL}$ of ethanol. The mixed solution was dried at $70{ }^{\circ} \mathrm{C}$, and was placed in a porcelain boat and heated to $600{ }^{\circ} \mathrm{C}$ for $240 \mathrm{~min}$ with a heating rate of $3{ }^{\circ} \mathrm{C} \cdot \mathrm{min}^{-1}$ under Ar atmosphere. The obtained sample was denoted as Ni SAC.

Electrochemical measurements. The electrochemical measurements were conducted on an electrochemical workstation (CH Instruments, $\mathrm{CHI}$ 660) in a three-electrode flow cell with $14 \mathrm{~mL}$ of $0.1 \mathrm{M} \mathrm{KHCO}_{3}$ solution ( $\mathrm{pH}$ 9) as the electrolyte. Gas diffusion layers (GDL) painted with $\mathrm{IrO}_{2}$ $\left(1 \mathrm{mg} \cdot \mathrm{cm}^{-2}\right)$ and catalysts $\left(1 \mathrm{mg} \cdot \mathrm{cm}^{-2}\right)$ were employed as the counter and working electrodes, respectively. $\mathrm{An} \mathrm{Ag} / \mathrm{AgCl}(3 \mathrm{M} \mathrm{KCl})$ electrode was used as the reference electrode. The gaseous and liquid products of CO reduction were detected by a gas chromatograph (Shanghai Ramiin GC 2060) and ${ }^{1} \mathrm{H}$ NMR on Bruker AVANCEAV III HD 500, respectively.

Typically, the electrochemical surface areas (ECSAs) and electrochemical impedance spectroscopy (EIS) measurements were conducted in a three-electrode system with $0.1 \mathrm{M} \mathrm{KHCO}_{3}$ solution ( $\mathrm{pH} 9$ ) as the electrolyte. A glassy carbon electrode (3-mm-diameter) was employed as the working electrode. A Pt wire and an $\mathrm{Ag} / \mathrm{AgCl}(3 \mathrm{M} \mathrm{KCl})$ electrode were used as the counter and reference electrodes, respectively. $5 \mathrm{mg}$ catalyst was dispersed in $1 \mathrm{~mL}$ acetone, and $60 \mu \mathrm{L}$ of $5 \mathrm{wt} \%$ Nafion was added subsequently. Afterwards, $20 \mu \mathrm{L}$ of the mixed solution was dripped on the surface of a glassy carbon electrode. 
Density functional theory (DFT) calculations. The DFT calculations were performed with the Vienna ab initio simulation package (VASP). The PBE functional with projector augmented wave $($ PAW) method was used. The modeled graphene supercell was $6 \times 6$, and some carbon atoms were removed to create the $\mathrm{Cu}-\mathrm{Cu}-\mathrm{N}_{6}$ and $\mathrm{Cu}-\mathrm{Ni}-\mathrm{N}_{6}$ motifs. A vacuum layer of $20 \AA$ was applied in order to avoid the interactions between periodic layers. The k-point mesh was $3 \times 3 \times 1$. A converged cutoff was set to $500 \mathrm{eV}$ for the expansion of wavefunctions over the plane-wave basis set. Considering the magnetism of $\mathrm{Ni}$, spin-polarization was included. In geometry optimization, configurations are relaxed with the force convergence $<0.02 \mathrm{eV} \cdot \AA^{-1}$. 


\section{Supplementary Figures}
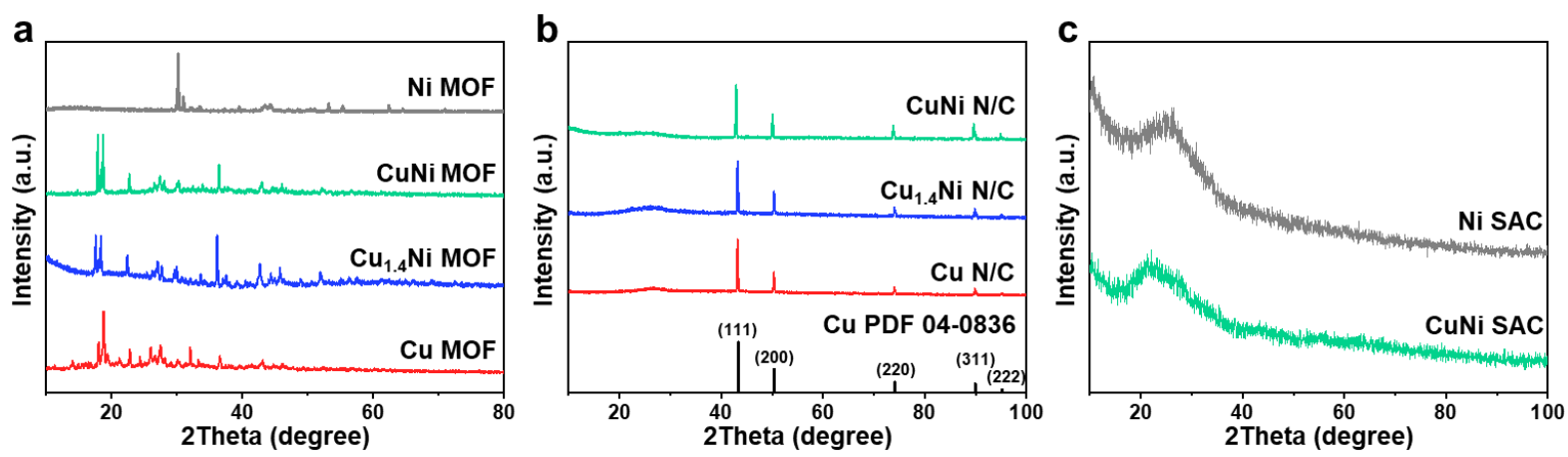

Figure S1. X-ray diffraction (XRD) characterization of different samples. (a) $\mathrm{Cu} \mathrm{MOF}, \mathrm{Cu}_{1.4} \mathrm{Ni}$ MOF, CuNi MOF, and Ni MOF. (b) $\mathrm{Cu}$ N/C, $\mathrm{Cu}_{1.4} \mathrm{Ni} / \mathrm{C}$, and $\mathrm{CuNi} / \mathrm{C}$. (c) CuNi SAC and Ni SAC.

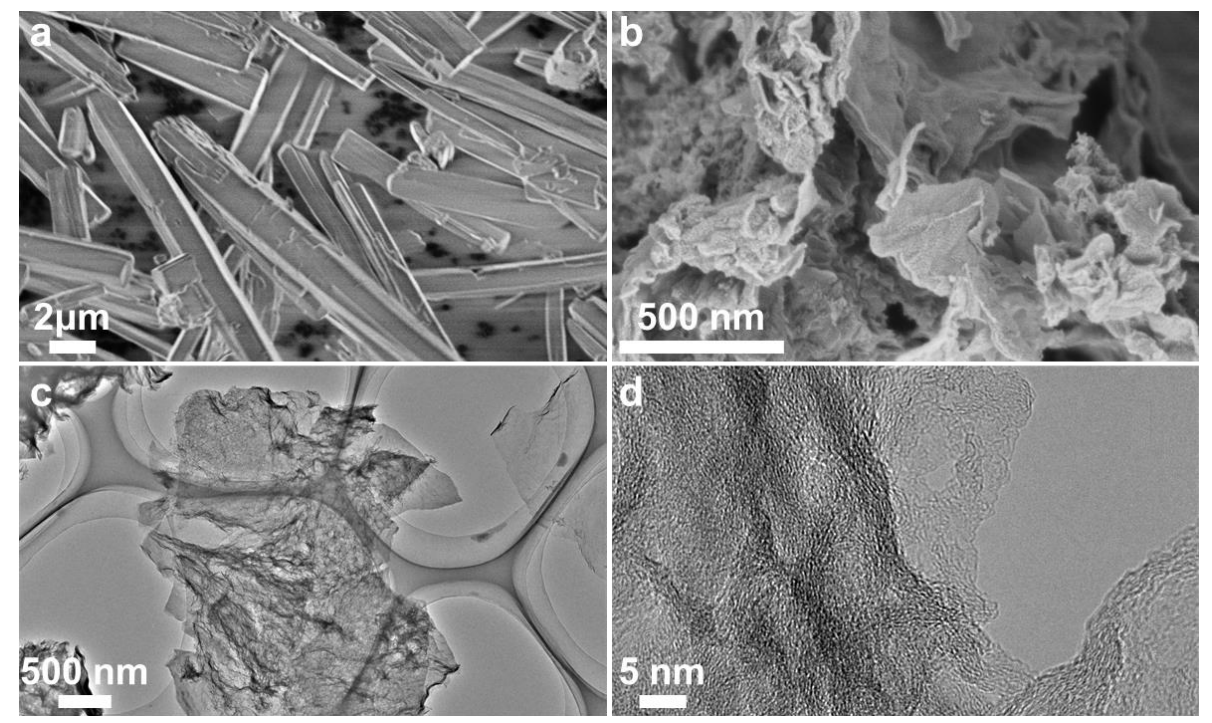

Figure S2. (a, b) SEM images of (a) Cu MOF and (b) Cu SAC. (c, d) TEM images of Cu SAC. 


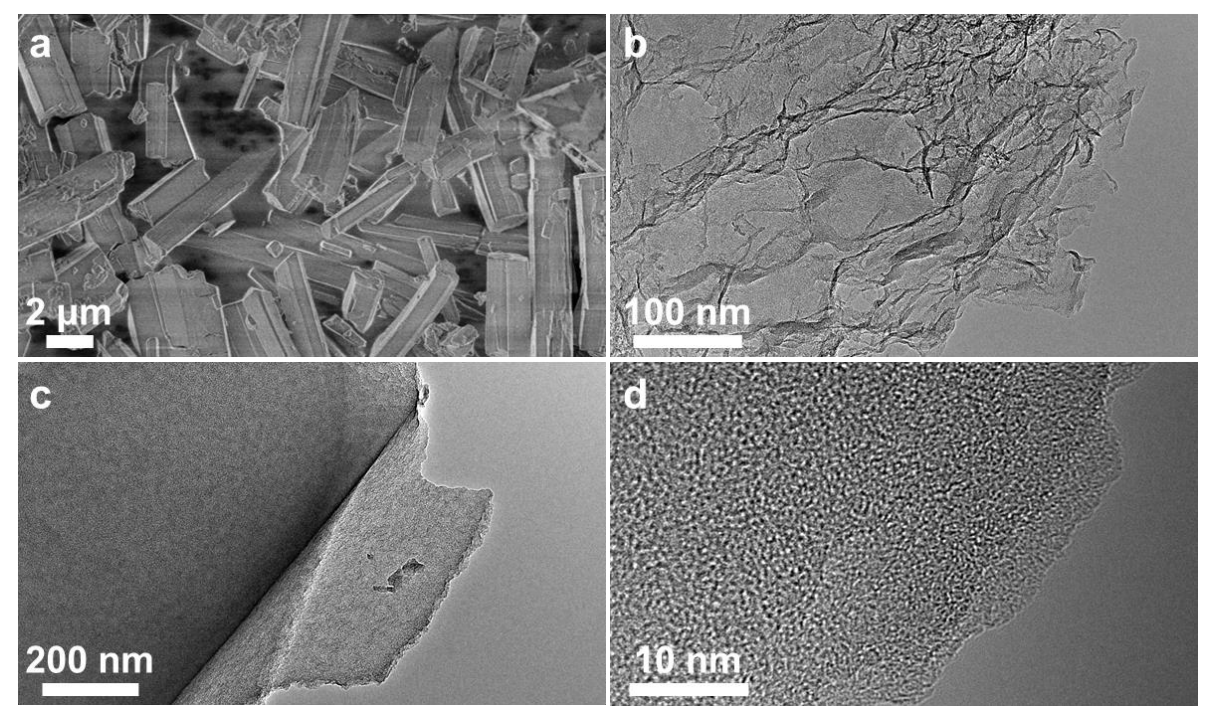

Figure S3. (a) SEM images of $\mathrm{Cu}_{1.4} \mathrm{Ni}$ MOF. TEM images of (b) $\mathrm{Cu}_{1.4} \mathrm{Ni}$ SAC, and (c, d) Ni SAC.

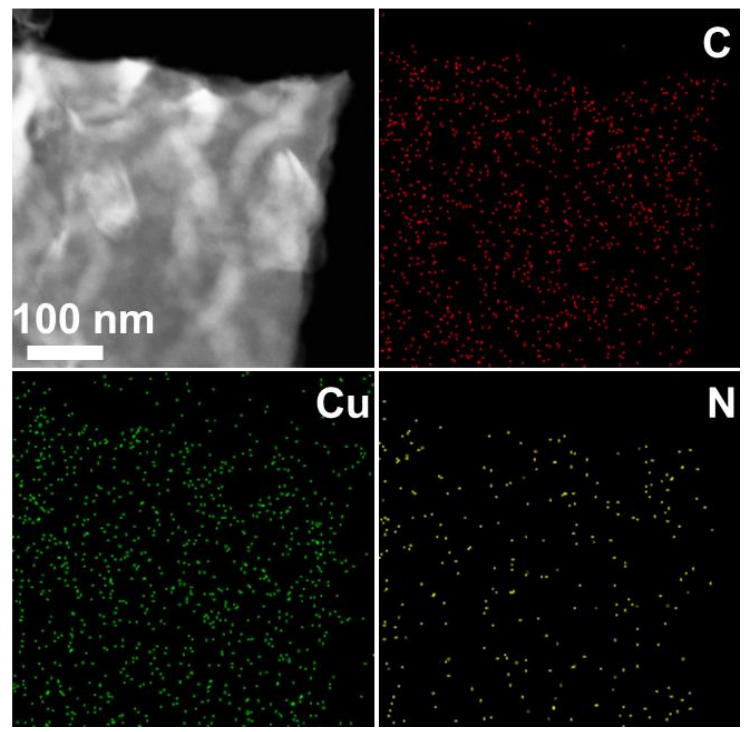

Figure S4. HADDF-STEM images and corresponding elemental mappings of Cu SAC. 

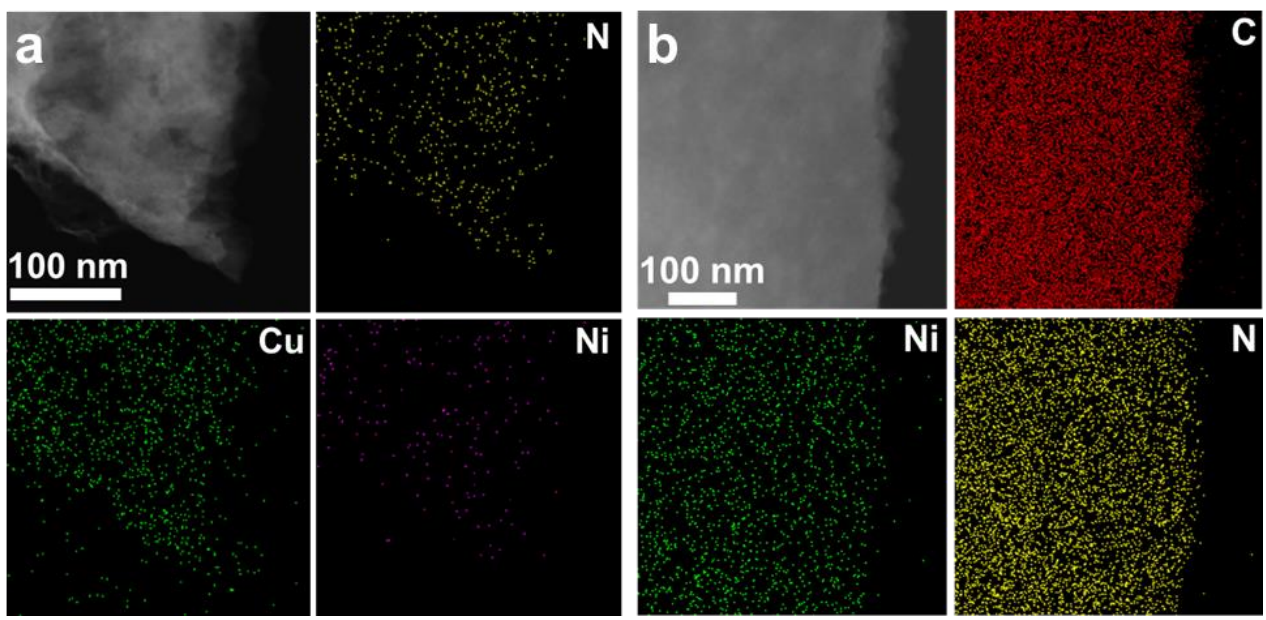

Figure S5. HADDF-STEM images and corresponding elemental mappings of (a) $\mathrm{Cu}_{1.4} \mathrm{Ni}$ SAC, and (b) Ni SAC.
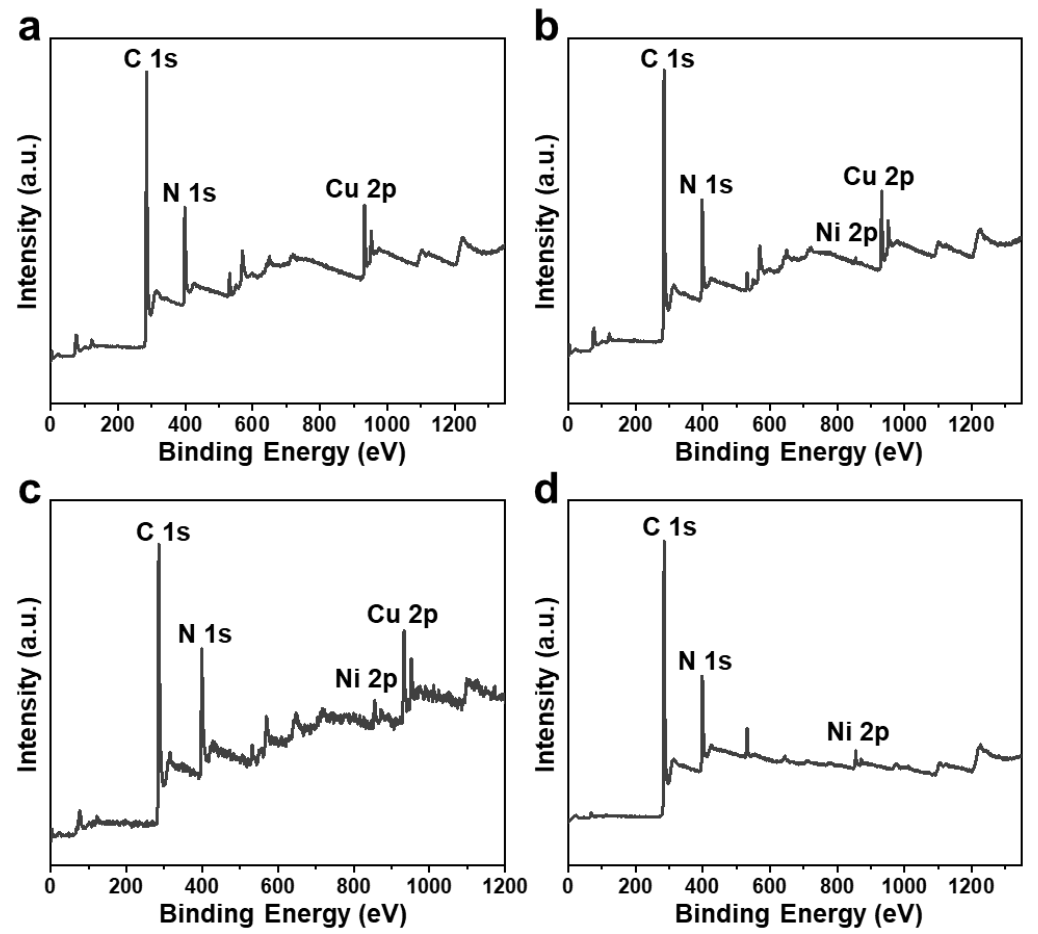

Figure S6. XPS survey spectra of (a) $\mathrm{Cu} S A C$, (b) $\mathrm{Cu}_{1.4} \mathrm{Ni} \mathrm{SAC}$, (c) CuNi SAC, and (d) Ni SAC. 

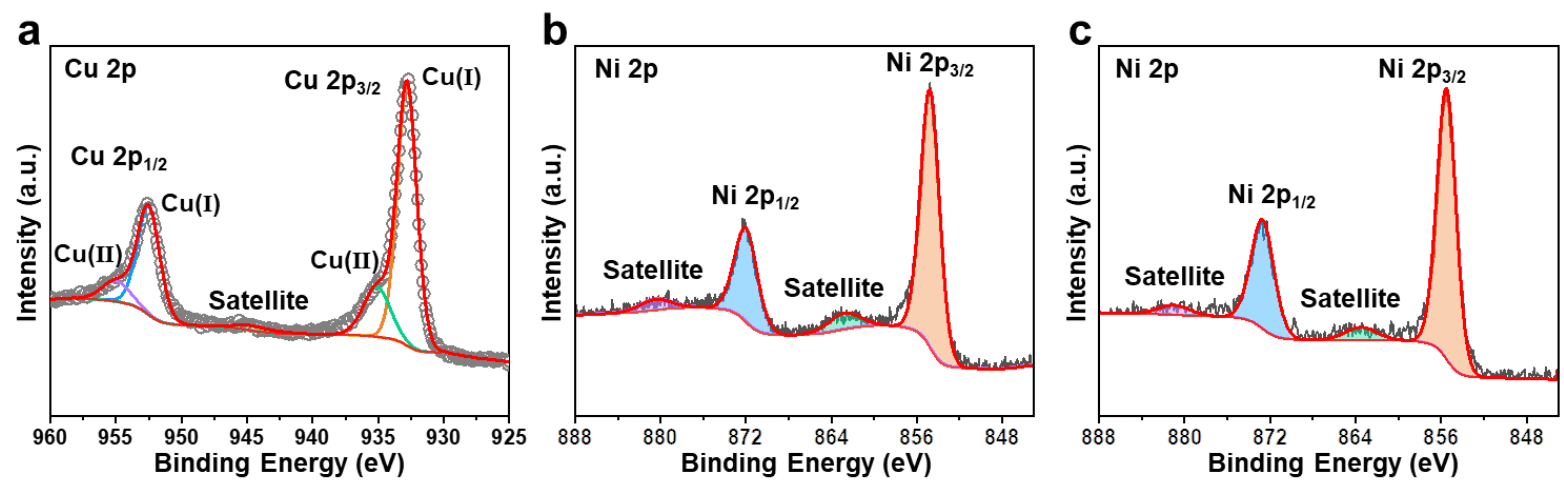

Figure S7. (a) Cu 2p XPS spectrum of CuNi SAC, (b) Ni 2p XPS spectrum of Ni SAC, and (c) Ni $2 p$ XPS spectrum of CuNi SAC.
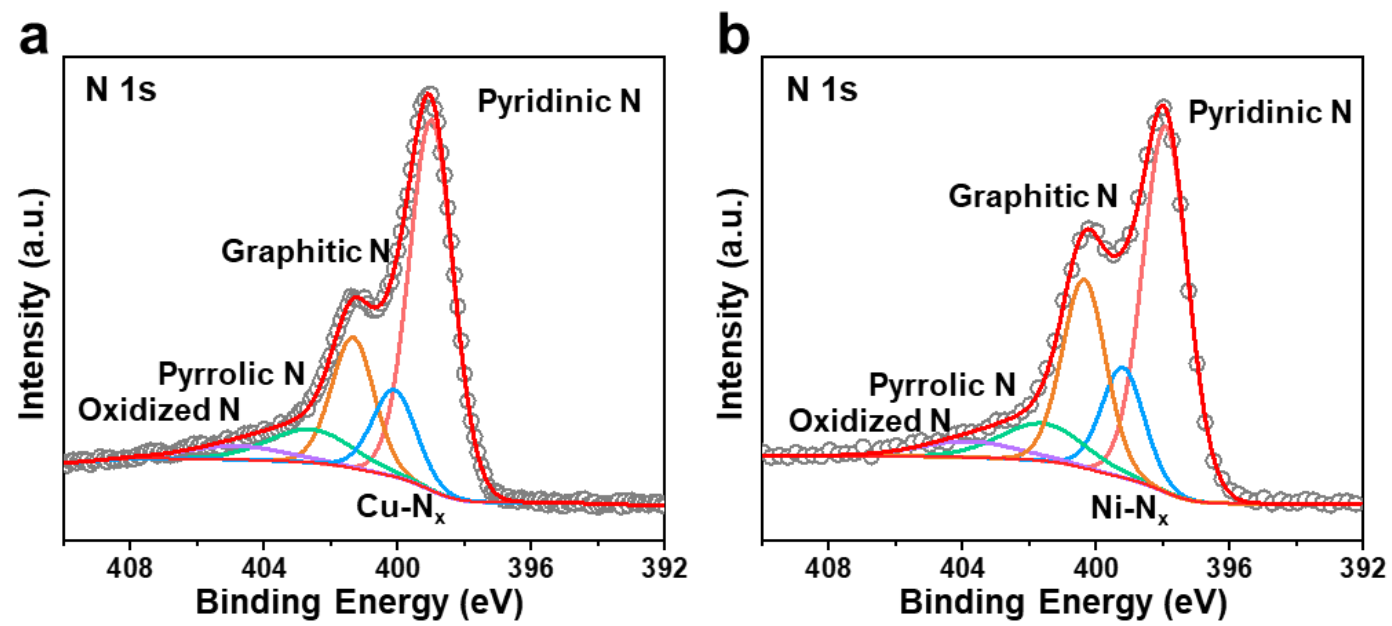

Figure S8. N 1s XPS spectra of (a) CuNi SAC, and (b) Ni SAC. 

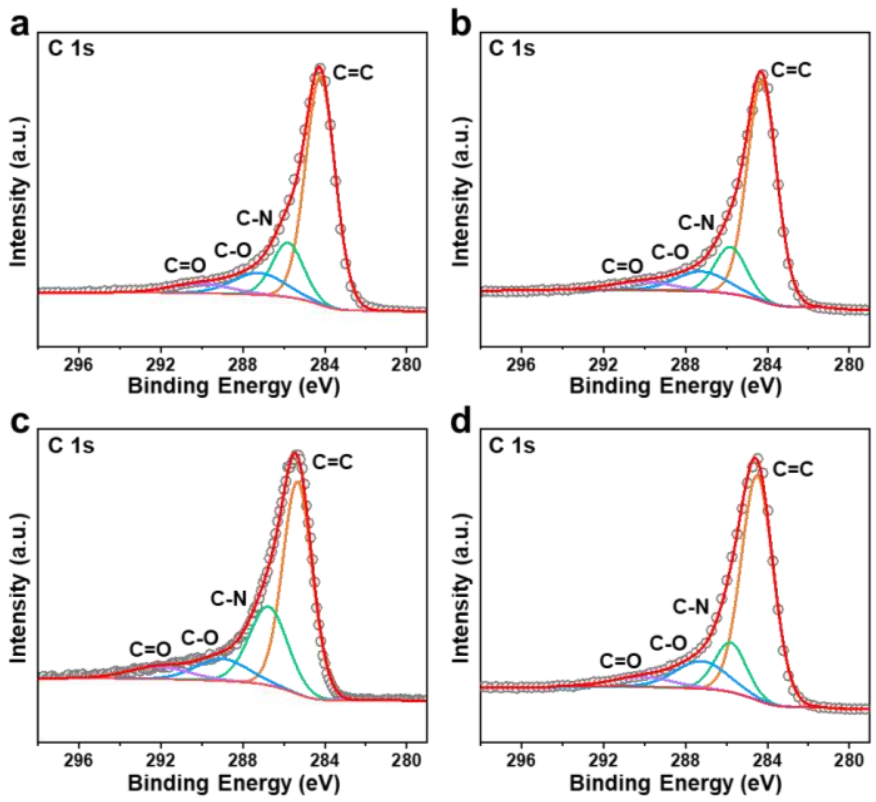

Figure S9. C 1s XPS spectra of (a) $\mathrm{Cu}$ SAC, (b) $\mathrm{Cu}_{1.4} \mathrm{Ni} \mathrm{SAC}$, (c) CuNi SAC, and (d) Ni SAC.
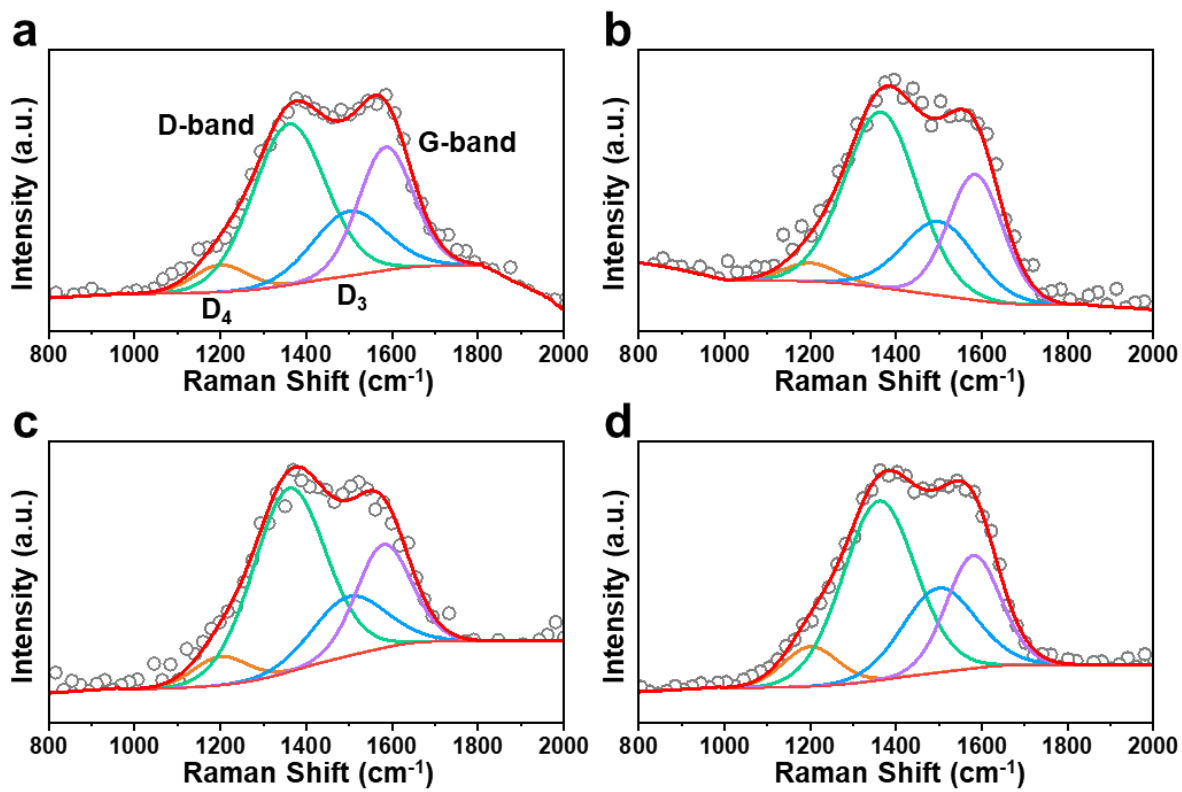

Figure S10. Raman spectra of (a) Cu SAC, (b) $\mathrm{Cu}_{1.4} \mathrm{Ni} \mathrm{SAC}$, (c) CuNi SAC, and (d) Ni SAC. 

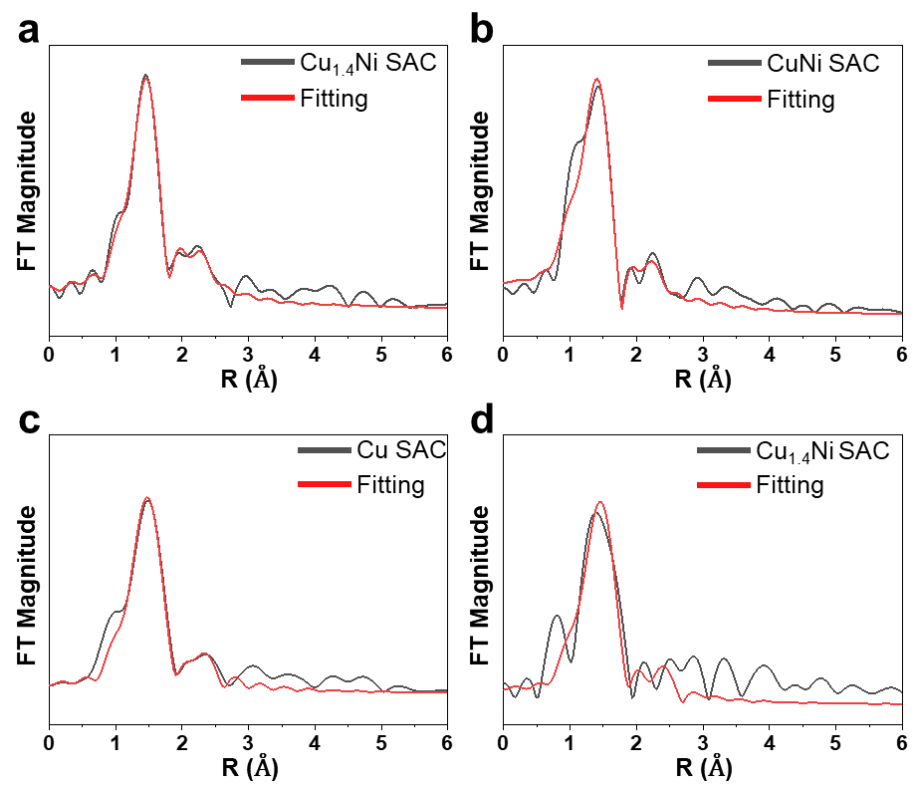

Figure S11. (a, b) Ni K-edge EXAFS fittings of (a) $\mathrm{Cu}_{1.4} \mathrm{Ni}$ SAC, and (b) CuNi SAC. (c, d) Cu Kedge EXAFS fittings of (c) Cu SAC and (b) $\mathrm{Cu}_{1.4} \mathrm{Ni}$ SAC.
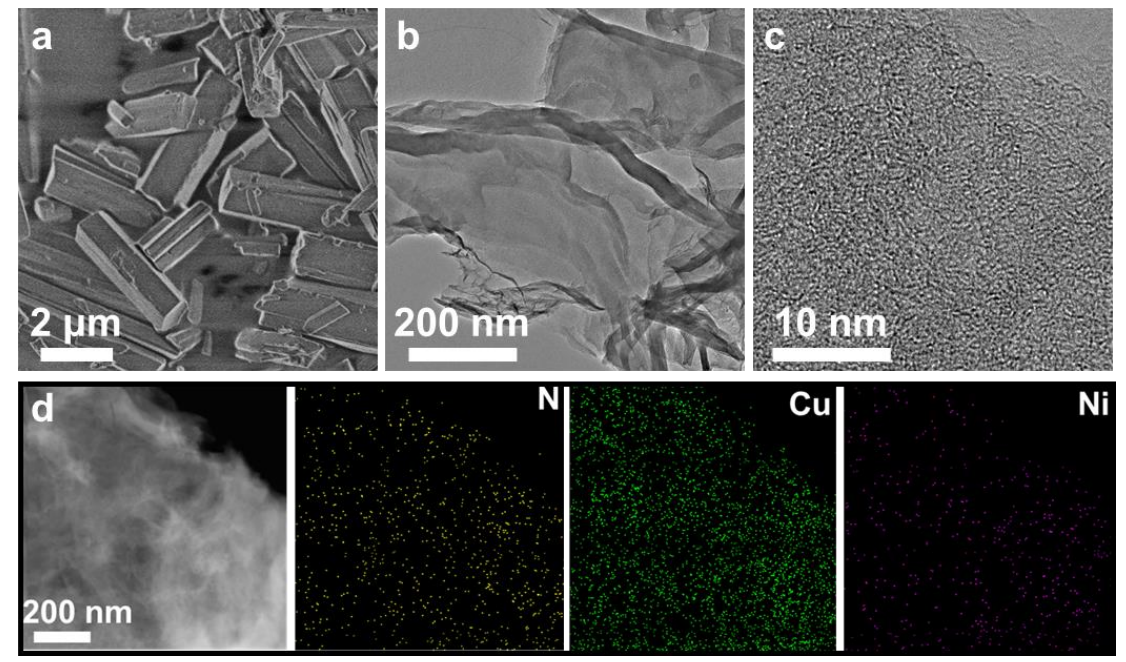

Figure S12. (a) SEM images of CuNi MOF, (b, c) TEM images of CuNi SAC, and (d) HADDFSTEM images and corresponding elemental mappings of CuNi SAC. 


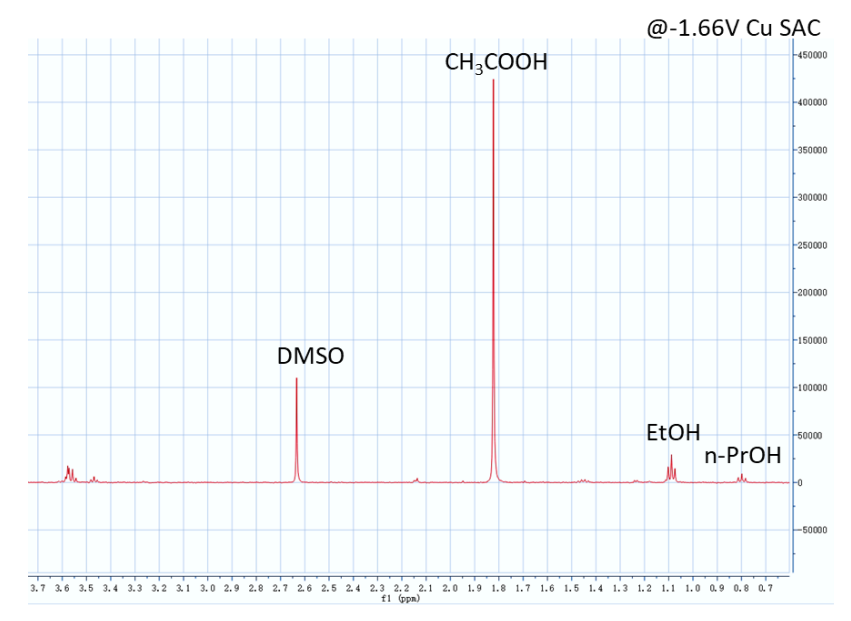

@-1.86V $\mathrm{Cu}_{1.4} \mathrm{Ni} \mathrm{SAC}$

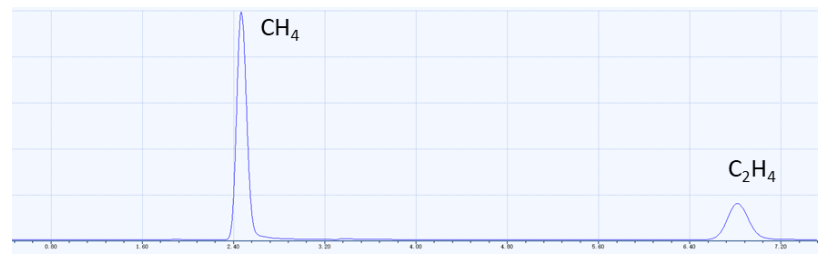

Figure S13. ${ }^{1} \mathrm{H}-\mathrm{NMR}$ data of the electrochemical reaction products of $\mathrm{Cu}$ SAC (upper panel) and gas chromatography results of $\mathrm{Cu}_{1.4} \mathrm{Ni}$ SAC (lower panel).

a

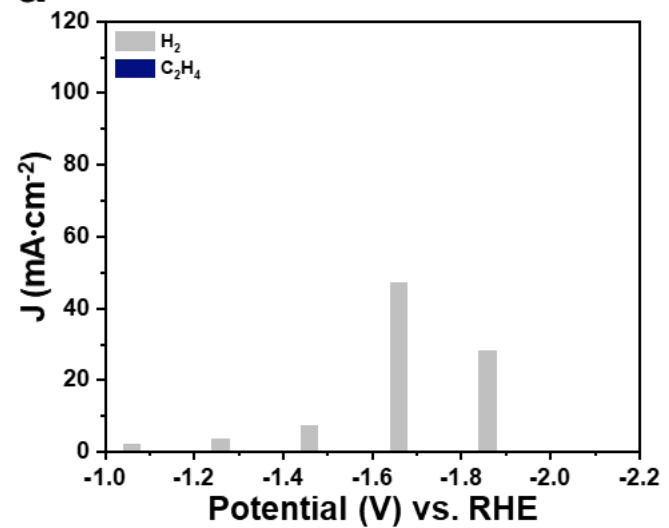

b

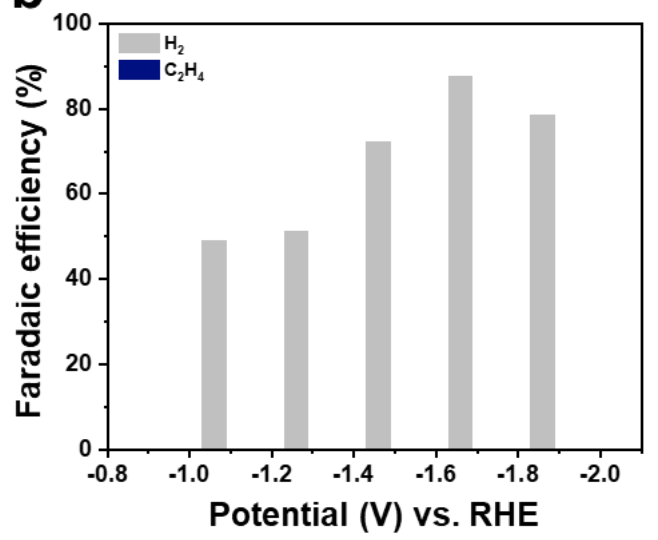

Figure S14. (a) Partial current densities and (b) Faradaic efficiency for different products on Ni SAC at different working potentials. 


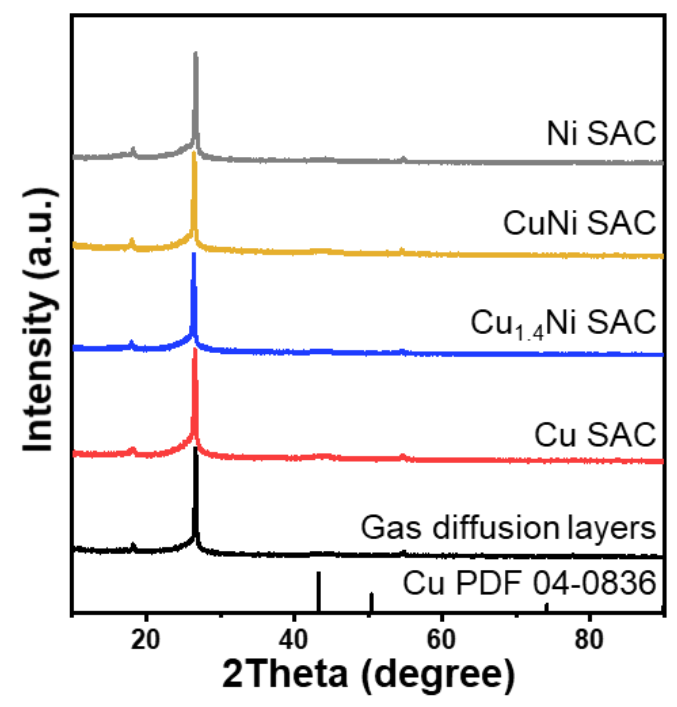

Figure S15. X-ray diffraction (XRD) characterization of different catalysts after CO electroreduction. 

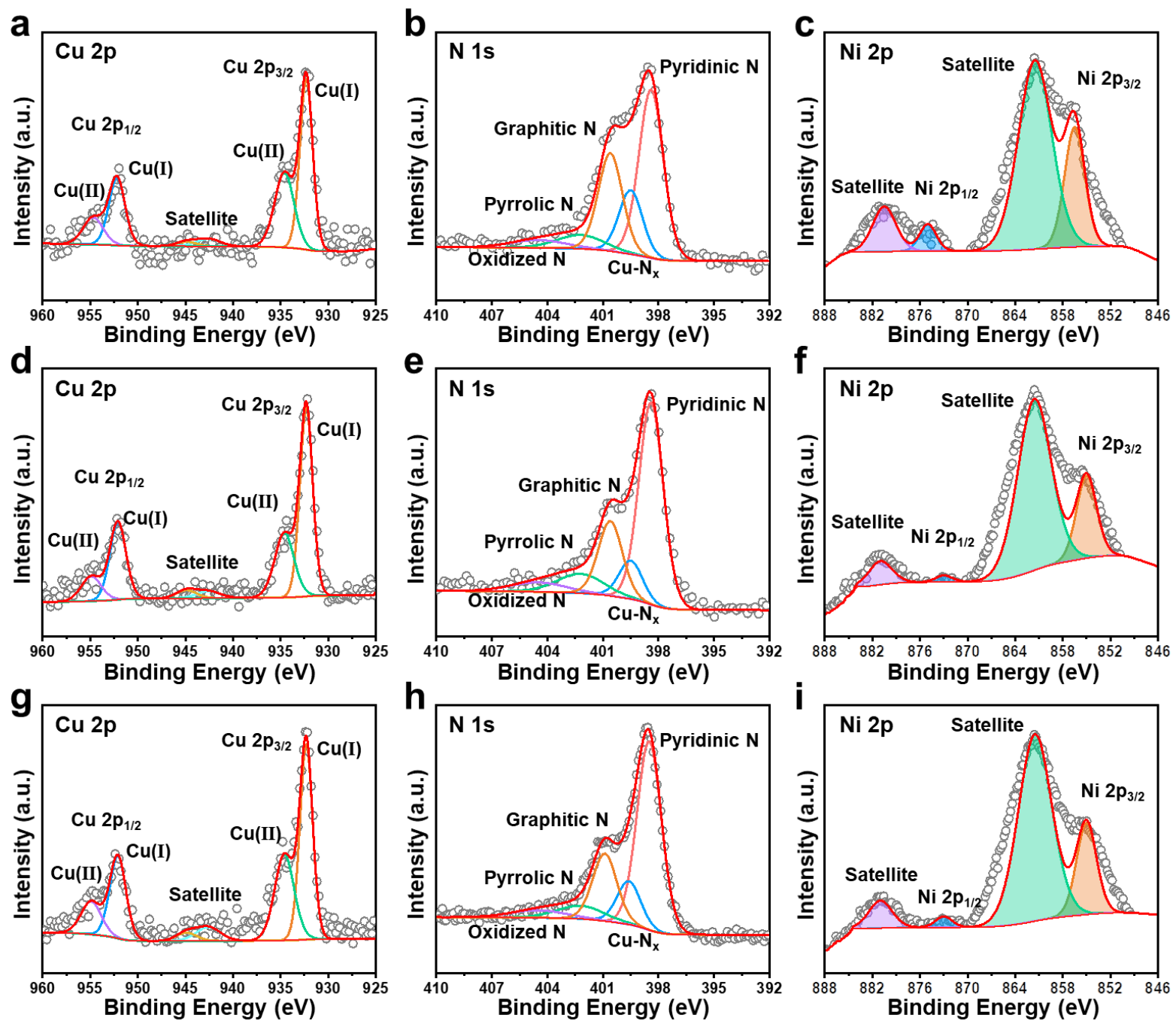

Figure S16. XPS characterization of different catalysts after $\mathrm{CO}$ electroreduction. (a) $\mathrm{Cu} 2 \mathrm{p}$ and

(b) N 1s XPS spectrum of Cu SAC. (c) Ni 2p XPS spectrum of Ni SAC. (d) Cu 2p, (e) N 1s and (f) $\mathrm{Ni} 2 \mathrm{p}$ XPS spectrum of $\mathrm{Cu}_{1.4} \mathrm{Ni}$ SAC. (g) $\mathrm{Cu} 2 \mathrm{p}$, (h) $\mathrm{N} 1 \mathrm{~s}$ and (i) $\mathrm{Ni} 2 \mathrm{p}$ XPS spectrum of CuNi SAC. 

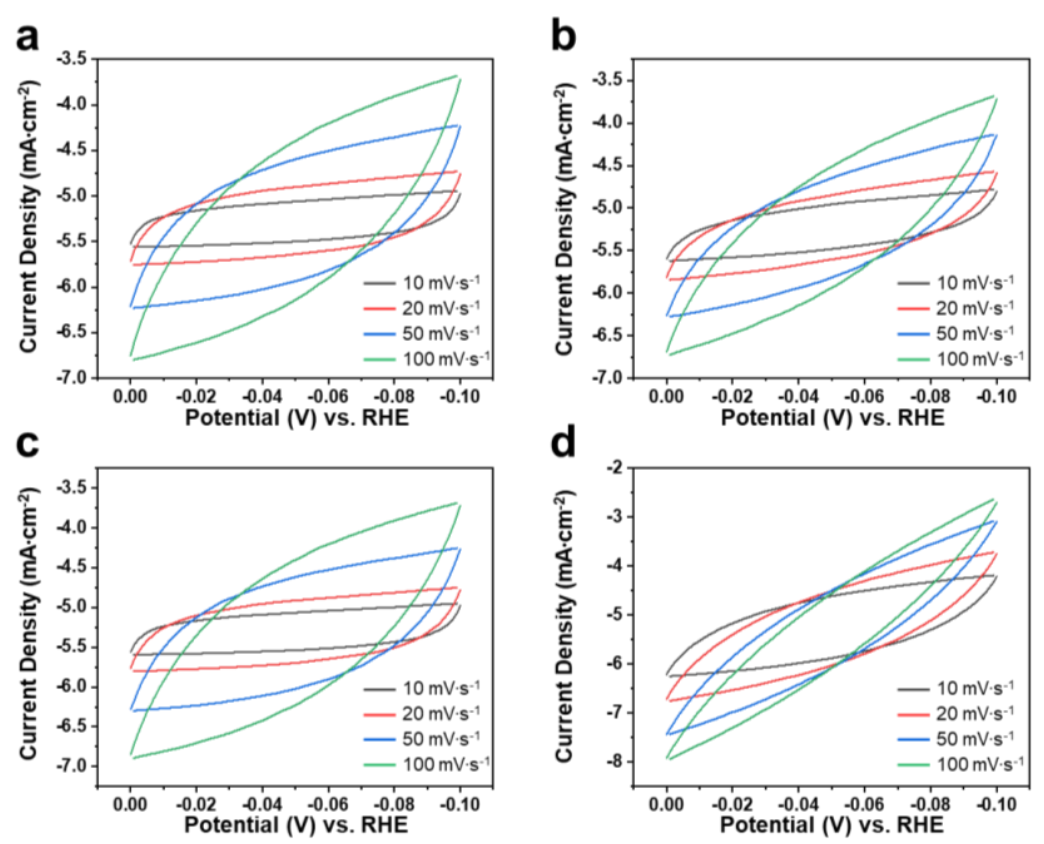

Figure S17. CV curves at various scan rates from 10 to $100 \mathrm{mV} \cdot \mathrm{s}^{-1}$ of (a) $\mathrm{Cu} \mathrm{SAC}$, (b) $\mathrm{Cu}_{1.4} \mathrm{Ni}$ SAC, (c) CuNi SAC, and (d) Ni SAC.

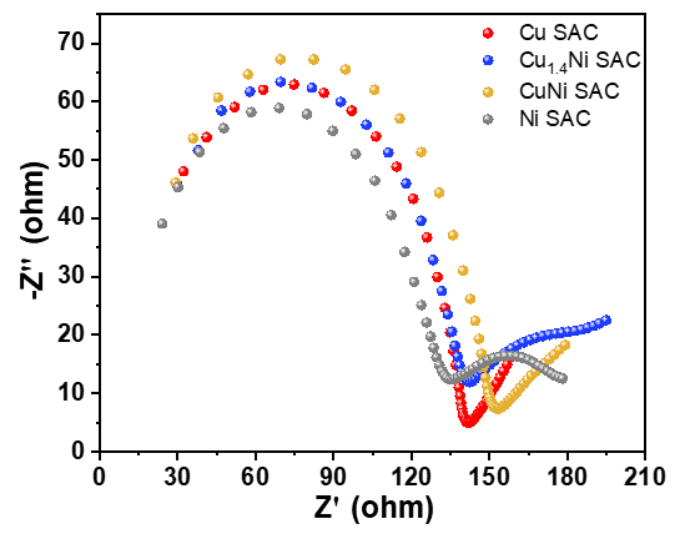

Figure S18. Electrochemical impedance spectroscopy measurements of $\mathrm{Cu} \mathrm{SAC}, \mathrm{Cu}_{1.4} \mathrm{Ni} \mathrm{SAC}$, CuNi SAC, and Ni SAC. 


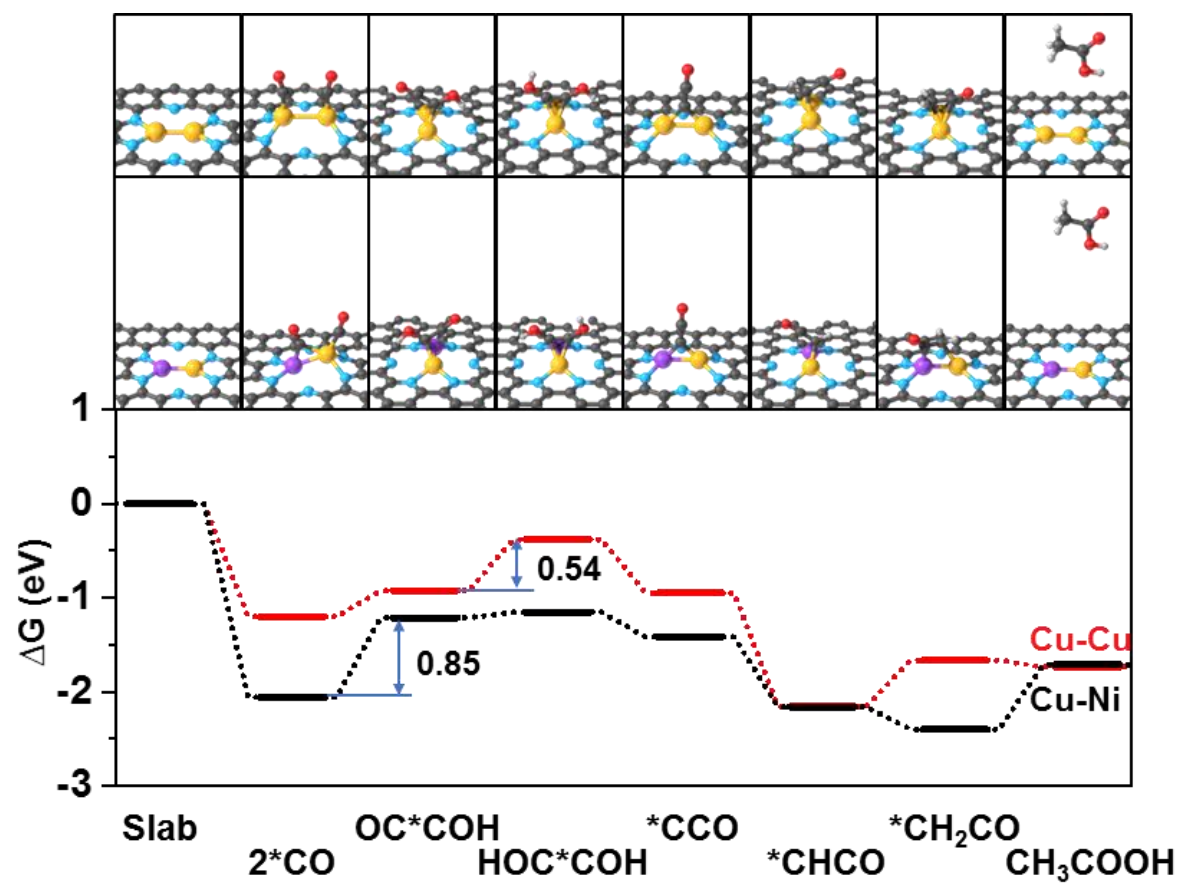

Figure S19. Gibbs free energy diagram for $\mathrm{CORR}$ (the path to $\mathrm{CH}_{3} \mathrm{COOH}$ ) on $\mathrm{Cu}-\mathrm{Cu} \mathrm{SAC}$ and $\mathrm{Cu}-\mathrm{Ni} \mathrm{SAC}$ at $\mathrm{U}=0 \mathrm{~V}$. Top panel: the path to $\mathrm{CH}_{3} \mathrm{COOH}$ on $\mathrm{Cu}-\mathrm{Cu}$ SAC. Down panel: the path to $\mathrm{CH}_{3} \mathrm{COOH}$ on $\mathrm{Cu}-\mathrm{Ni} \mathrm{SAC}$. 
Table S1. $\mathrm{Cu}$ and Ni contents in $\mathrm{Cu}-\mathrm{Ni}$ based samples tested by XPS.

\begin{tabular}{ccc}
\hline Sample & Element & $\begin{array}{c}\text { atomic percent } \\
\text { contents } / \%\end{array}$ \\
\hline $\mathrm{Cu} \mathrm{SAC}$ & $\mathrm{Cu}$ & 2.26 \\
$\mathrm{Cu}_{1.4} \mathrm{NiSAC}$ & $\mathrm{Ni}$ & 1.81 \\
& $\mathrm{Cu}$ & 2.54 \\
\hline $\mathrm{CuNiSAC}$ & $\mathrm{Ni}$ & 2.49 \\
\hline $\mathrm{NiSAC}$ & $\mathrm{Cu}$ & 2.52 \\
\hline
\end{tabular}

Table S2. $\mathrm{C}$ and $\mathrm{N}$ contents in $\mathrm{Cu}-\mathrm{Ni}$ based samples tested by XPS.

\begin{tabular}{ccc}
\hline Sample & Element & $\begin{array}{c}\text { atomic percent } \\
\text { contents } / \%\end{array}$ \\
\hline $\mathrm{Cu}$ SAC & $\mathrm{C}$ & 72.75 \\
& $\mathrm{~N}$ & 8.62 \\
\hline $\mathrm{Cu}_{1.4} \mathrm{NiSAC}$ & $\mathrm{C}$ & 72.68 \\
& $\mathrm{~N}$ & 12.97 \\
\hline $\mathrm{CuNiSAC}$ & $\mathrm{C}$ & 72.91 \\
& $\mathrm{~N}$ & 12.24 \\
\hline $\mathrm{NiSAC}$ & $\mathrm{C}$ & 74.03 \\
& $\mathrm{~N}$ & 6.98 \\
\hline
\end{tabular}


Table S3. Structural parameters of $\mathrm{Cu}$ SAC, $\mathrm{Cu}_{1.4} \mathrm{Ni}$ SAC, and CuNi SAC obtained from EXAFS fittings.

\begin{tabular}{|c|c|c|c|c|c|c|c|c|}
\hline sample & Structure & $\begin{array}{c}\text { Scattering } \\
\text { path }\end{array}$ & $\mathbf{R}(\AA)$ & $\mathbf{C N}$ & $\sigma^{2}$ & $\begin{array}{c}E_{0} \\
\text { shift } \\
(\mathrm{eV})\end{array}$ & $\mathrm{S}_{0}{ }^{2}$ & $\begin{array}{c}\text { R- } \\
\text { factor }\end{array}$ \\
\hline \multirow{2}{*}{$\begin{array}{c}\mathrm{Cu}_{1.4} \mathrm{Ni} \\
\mathrm{SAC}\end{array}$} & $\mathrm{Cu}_{3} \mathrm{Ni}$ & $\mathrm{Ni}-\mathrm{Cu}$ & $2.306 \pm 0.033$ & 4 & 0.020 & 8.152 & 0.9 & 0.006 \\
\hline & $\mathrm{Ni}_{3} \mathrm{~N}$ & $\mathrm{Ni}-\mathrm{N}$ & $1.857 \pm 0.006$ & 2 & 0.002 & 8.152 & 0.9 & 0.006 \\
\hline \multirow{2}{*}{$\begin{array}{l}\mathrm{CuNi} \\
\mathrm{SAC}\end{array}$} & $\mathrm{Cu}_{3} \mathrm{Ni}$ & $\mathrm{Ni}-\mathrm{Cu}$ & $2.319 \pm 0.059$ & 4 & 0.025 & $\overline{-} . \overline{358}$ & 0.9 & 0.011 \\
\hline & $\mathrm{Ni}_{3} \mathrm{~N}$ & $\mathrm{Ni}-\mathrm{N}$ & $1.871 \pm 0.013$ & 2 & 0.002 & $\begin{array}{c}- \\
2.358\end{array}$ & 0.9 & 0.011 \\
\hline \multirow{2}{*}{$\begin{array}{l}\mathrm{Cu} \\
\mathrm{SAC}\end{array}$} & $\mathrm{Cu}$ & $\mathrm{Cu}-\mathrm{Cu}$ & $2.579 \pm 0.079$ & 12 & 0.005 & 5.859 & 0.9 & 0.007 \\
\hline & $\mathrm{Cu}_{3} \mathrm{~N}$ & $\mathrm{Cu}-\mathrm{N}$ & $1.911 \pm 0.013$ & 2 & 0.008 & 5.859 & 0.9 & 0.007 \\
\hline \multirow{3}{*}{$\begin{array}{c}\mathrm{Cu}_{1.4} \mathrm{Ni} \\
\mathrm{SAC}\end{array}$} & $\mathrm{Cu}_{3} \mathrm{Ni}$ & $\mathrm{Cu}-\mathrm{Ni}$ & $2.807 \pm 0.068$ & 2 & 0.010 & 3.117 & 0.9 & 0.020 \\
\hline & $\mathrm{Cu}_{3} \mathrm{Ni}$ & $\mathrm{Cu}-\mathrm{Cu}$ & $2.866 \pm 0.068$ & 2 & 0.010 & 3.117 & 0.9 & 0.020 \\
\hline & $\mathrm{Cu}_{3} \mathrm{~N}$ & $\mathrm{Cu}-\mathrm{N}$ & $1.908 \pm 0.015$ & 2 & 0.009 & 3.117 & 0.9 & 0.020 \\
\hline
\end{tabular}


Table S4. Comparison of different Cu-based catalysts in CORR.

\begin{tabular}{|c|c|c|c|c|c|c|c|}
\hline \multirow{2}{*}{ Catalyst } & \multicolumn{3}{|c|}{ Faradaic efficiency (\%) } & \multirow{2}{*}{ Electrolyte } & \multirow{2}{*}{ Device } & \multirow{2}{*}{$\begin{array}{c}\text { Current } \\
\text { density } \\
\left(\mathbf{m A} \cdot \mathbf{c m}^{-2}\right)\end{array}$} & \multirow{2}{*}{ Reference } \\
\hline & $\mathrm{CH}_{4}$ & $\mathbf{H}_{2}$ & $\mathrm{C}_{2+\text { products }}$ & & & & \\
\hline $\mathrm{Cu} \mathrm{SAC}$ & $\sim 5$ & $\sim 10$ & $\sim 90$ & $0.1 \mathrm{M} \mathrm{KHCO}_{3}$ & Flow cell & $\sim 100$ & Our work \\
\hline $\mathrm{Cu}$ nanoparticle & l & I & 18 & $10 \mathrm{M} \mathrm{KOH}$ & Flow cell & $\sim 300$ & {$[\mathrm{~S} 1]$} \\
\hline oxide-derived $\mathrm{Cu}$ & I & $\sim 10$ & $\sim 90$ & $2 \mathrm{M} \mathrm{KOH}$ & Flow cell & $\sim 1000$ & {$[\mathrm{~S} 2]$} \\
\hline $\mathrm{Cu}$ nanosheets & 2.3 & 15.8 & $\sim 69$ & $2 \mathrm{M} \mathrm{KOH}$ & Flow cell & 200 & {$[\mathrm{~S} 3]$} \\
\hline $\mathrm{Cu}$ sheets & 22.3 & 41.7 & 32.9 & $0.1 \mathrm{M} \mathrm{KHCO}_{3}$ & H-cell & 2.5 & {$[\mathrm{~S} 4]$} \\
\hline oxide-derived $\mathrm{Cu}$ & / & / & 48 & $0.1 \mathrm{M} \mathrm{KOH}$ & H-cell & $0.01-0.001$ & {$[\mathrm{~S} 5]$} \\
\hline oxide-derived $\mathrm{Cu}$ & / & / & 57.1 & $0.1 \mathrm{M} \mathrm{KOH}$ & H-cell & 0.285 & {$[\mathrm{~S} 6]$} \\
\hline $\begin{array}{c}\mathrm{Cu} \\
\text { nanoparticles/carbo } \\
\mathrm{n} \text { nanotubes } \\
\end{array}$ & / & l & $\sim 70$ & $0.1 \mathrm{M} \mathrm{KOH}$ & H-cell & 0.37 & [S7] \\
\hline $\mathrm{Cu}$ nanowires & l & I & $\sim 65$ & $0.1 \mathrm{M} \mathrm{KOH}$ & H-cell & $\sim 0.5$ & {$[\mathrm{~S} 8]$} \\
\hline $\begin{array}{l}\text { Three-Dimensional } \\
\text { Nanostructured } \mathrm{Cu}\end{array}$ & l & / & 76 & $0.1 \mathrm{M} \mathrm{KOH}$ & H-cell & 6.9 & [S9] \\
\hline $\begin{array}{l}\text { Polycrystalline } \\
\qquad \mathrm{Cu}\end{array}$ & 0 & 35.8 & 52.6 & $0.1 \mathrm{M} \mathrm{KOH}$ & H-cell & 1 & {$[\mathrm{~S} 10]$} \\
\hline $\mathrm{Cu}$ films & $\sim 1$ & 61.6 & $\sim 40$ & $\begin{array}{l}0.05 \mathrm{M} \\
\mathrm{KHCO}_{3}\end{array}$ & H-cell & 1.56 & {$[\mathrm{~S} 11]$} \\
\hline Polycrystalline $\mathrm{Cu}$ & $\sim 3$ & $\sim 35$ & $\sim 65$ & $1 \mathrm{M} \mathrm{NaOH}$ & H-cell & 25 & {$[\mathrm{~S} 12]$} \\
\hline
\end{tabular}




\section{References}

[S1] Han, L.; Zhou, W.; Xiang, C. High-Rate Electrochemical Reduction of Carbon Monoxide to Ethylene Using Cu-Nanoparticle-Based Gas Diffusion Electrodes. ACS Energy Lett. 2018, 3, 855-860.

[S2] Jouny, M.; Luc, W.; Jiao, F. High-Rate Electroreduction of Carbon Monoxide to MultiCarbon Products. Nat. Catal. 2018, 1, 748-755.

[S3] Luc, W.; Fu, X.; Shi, J.; Lv, J.; Jouny, M.; Ko, B.; Xu, Y.; Tu, Q.; Hu, X.; Wu, J.; Yue, Q.; Liu, Y.; Jiao, F.; Kang, Y. Two-Dimensional Copper Nanosheets for Electrochemical Reduction of Carbon Monoxide to Acetate. Nat. Catal. 2019, 2, 423-430.

[S4] Hori, Y.; Takahashi, R.; Yoshinami, Y.; Murata, A. Electrochemical Reduction of CO at a Copper Electrode. J. Phys. Chem. B 1997, 101, 7075-7081.

[S5] Verdaguer, C. A.; Li, C.; Johansson, T. P.; Scott, S. B.; McKeown, J. T.; Kumar, M.; Stephens, I. L.; Kanan, M. W.; Chorkendorff, I. Probing the Active Surface Sites for CO Reduction on Oxide-Derived Copper Electrocatalysts. J. Am. Chem. Soc. 2015, 137, 9808-9811.

[S6] Li, C.; Ciston, J.; Kanan, M. W. Electroreduction of Carbon Monoxide to Liquid Fuel on Oxide-Derived Nanocrystalline Copper. Nature 2014, 508, 504-507.

[S7] Feng, X.; Jiang, K.; Fan, S.; Kanan, M. A Direct Grain-Boundary-Activity Correlation for CO Electroreduction on Cu Nanoparticles. ACS Cent. Sci. 2016, 2, 169-174.

[S8] Raciti, D.; Cao, L.; Liv, K. T.; Rottmann, P. F.; Tang, X.; Li, C.; Hicks, Z.; Bowen, K. H.; Hemker, K. J.; Mueller, T.; Wang, C. Low-Overpotential Electroreduction of Carbon Monoxide Using Copper Nanowires. ACS Catal. 2017, 7, 4467-4472.

[S9] Wang, Y.; Raciti, D.; Wang, C. High-Flux CO Reduction Enabled by Three-Dimensional Nanostructured Copper Electrodes. ACS Catal. 2018, 8, 5657-5663.

[S10] Wang, L.; Nitopi, S. A.; Bertheussen, E.; Orazov, M.; Morales-Guio, C. G.; Liu, X.; Higgins, D. C.; Chan, K.; Norskov, J. K.; Hahn, C.; Jaramillo, T. F. Electrochemical Carbon Monoxide Reduction on Polycrystalline Copper: Effects of Potential, Pressure, 
and $\mathrm{pH}$ on Selectivity toward Multicarbon and Oxygenated Products. ACS Catal. 2018, $8,7445-7454$.

[S11] Lum, Y. W.; Cheng, T.; Goddard, W. A.; Ager, J. W. Electrochemical CO Reduction Builds Solvent Water into Oxygenate Products. J.Am. Chem. Soc. 2018, 140, 9337-9340.

[S12] Li, J.; Wu, D.; Malkani, A. S.; Chang, X.;Cheng, M.; Xu. B.; Lu, Q. Hydroxide is not a Promoter of $\mathrm{C}_{2+}$ Product Formation in the Electrochemical Reduction of $\mathrm{CO}$ on Copper. Angew. Chem., Int. Ed. 2020, 59, 4464-4469. 